\title{
IDENTIFICATION OF THE POST-MAXIMUM LINES IN THE SPECTRUM OF NOVA (RS) OPHIUCHI*
}

\author{
A. H. Jox and P. Swings \\ Mount Wilson Observatory \\ Received August 6, 1945
}

\section{ABSTRACT}

Interest in the spectra of recurring novae has been aroused by the recent (fourth) outburst of Nova (T) Pyxidis. A review of the hitherto unpublished measures of the Mount Wilson spectrograms of Nova (RS) Ophiuchi obtained between September 2 and November 10,1933, shows many plausible identifications of lines which were impossible earlier.

The atoms now identified, in addition to $H$, are: $H e \mathrm{I}, H e \mathrm{II}, C \mathrm{III},[N \mathrm{~m}], N \mathrm{mr},[O \mathrm{I}], O \mathrm{III},[O \mathrm{II}]$, [Ne III], [Ne Iv], $N a$ I, $S i \mathrm{I}, S i \mathrm{II}, S i \mathrm{III}, S i \mathrm{IV},[S \mathrm{II}],[S \mathrm{III}],[A \mathrm{v}],[A \mathrm{x}],[A \mathrm{xI}],[K \mathrm{IV}],[K \mathrm{v}],[C a \mathrm{v}]$, [Ca vI], [Ca vII], [Ca XIII], [Sc VII], [V vII],$F e$ II, [Fe II], [Fe IV], [Fe v], [Fe vI], [Fe vII], $[F e \mathrm{x}],[F e \mathrm{XI}]$,

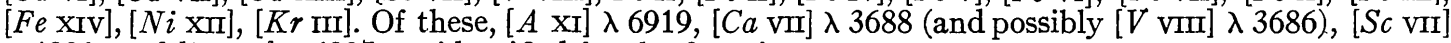
$\lambda 4823$, and $[K r$ III] $\lambda 6827$ are identified for the first time.

Considerations of the physical conditions in the solar corona as compared with those in the recurrent novae indicate marked differences, the coronal strata not permitting radiation of forbidden lines in the lower stages of ionization such as are found in the novae.

The maximum of the second outburst of Nova (RS) Ophiuchi, which occurred about August 12, 1933, showed spectral characteristics similar to those of other "fast" novae. Bright lines of $\mathrm{H}, \mathrm{He}, \mathrm{Fe} \mathrm{I}, \mathrm{Ca} \mathrm{I}$, and $\mathrm{Na}$ I, attaining a total width of about $25 \mathrm{~A}$ for the strongest lines, were observed from August 16 to September $11 .^{1}$ These lines did not have the strong absorption components usually found at the violet edge of the chief lines of novae spectra at this phase, but, with sufficient dispersion, they showed a narrow dark line slightly displaced toward the violet from the center of the bright line.

Comparatively sharp nebular lines soon appeared and were first observed as follows: $\lambda 4362[O \mathrm{III}]$ on August 18; $\lambda 5006$ [O III], $\lambda 4640 \mathrm{~N}$ III, and $\lambda 4686 \mathrm{He}$ II on August 29; $\lambda 3868$ [ $\mathrm{Ne}$ III],$\lambda 3967$ [Ne III], and $\lambda 4959$ [O III] on August 30; [ $\mathrm{Fe}$ II] on September 11; and $\lambda 4068[S \mathrm{II}]$ on October 1.

The coronal lines were first definitely identified ${ }^{2}$ on a spectrogram taken on October 2, but it seems probable that $\lambda 6374$ was present as early as September 7, enhancing the strength of $\lambda 6371 S i$ II above that of $\lambda 6347 S i$ II. The lines $\lambda 5303$ and $\lambda 6374$, previously observed only in the solar corona, were strong on October 2 and changed only slightly in intensity until the end of the observing season on November 10,1933. At the end of October, $\lambda 5303$ was of intensity comparable to that of $H \beta$, and $\lambda 6374$ was more than twice as strong as $\lambda 5875 \mathrm{He}$. By the beginning of the next observing season in March, 1934, the coronal lines had completely disappeared.

On the Mount Wilson spectrograms obtained between September 2 and November 10,1933 , after the first appearance of the nebular and coronal lines, a considerable number of lines was measured whose identity could not be recognized at that time. In the last few years our knowledge of the forbidden transitions has increased many fold, so that a new attempt to identify the lines which attained their greatest intensity some two months or more after the maximum of the nova now seems justified. The recent outburst of the recurrent Nova (T) Pyxidis and the satisfactory identification ${ }^{3}$ of nearly all its emission lines add new interest to the problem.

* Contributions from the Mount Wilson Observatory, Carnegie Institution of Washington, No. 714.

${ }^{1}$ Adams and Joy, Pub. A.S.P., 45, 249, 1933; Wright and Neubauer, Pub. A.S.P., 45, 252, 1933.

${ }^{2}$ Adams and Joy, Pub. A.S.P., 45, 301, 1933.

${ }^{3}$ A. H. Joy, Pub. A.S.P., 57, 171, 1945. The unidentified line $\lambda 7999$ in T Pyxidis may possibly be the $\mathrm{a}^{6} \mathrm{~S}_{3 \frac{1}{2}}-\mathrm{a}^{6} \mathrm{D}_{41 / 2}$ transition of $[\mathrm{Cr}$ II] whose predicted wave length is 8000.12 . 
TABLE 1

IDENTIFICATION OF EMISSION LINES IN RS OPHIUCHI

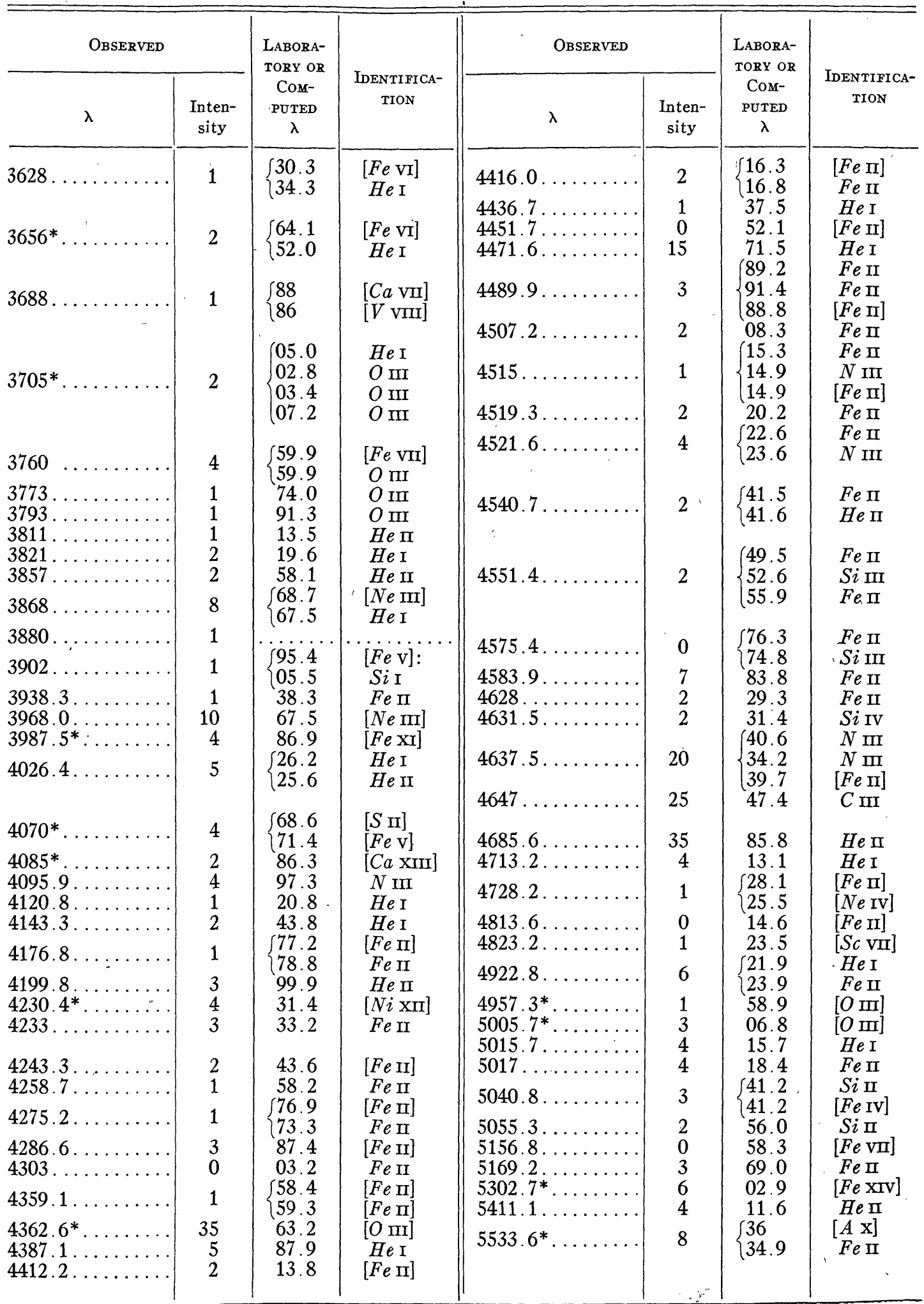


TABLE 1-Continued

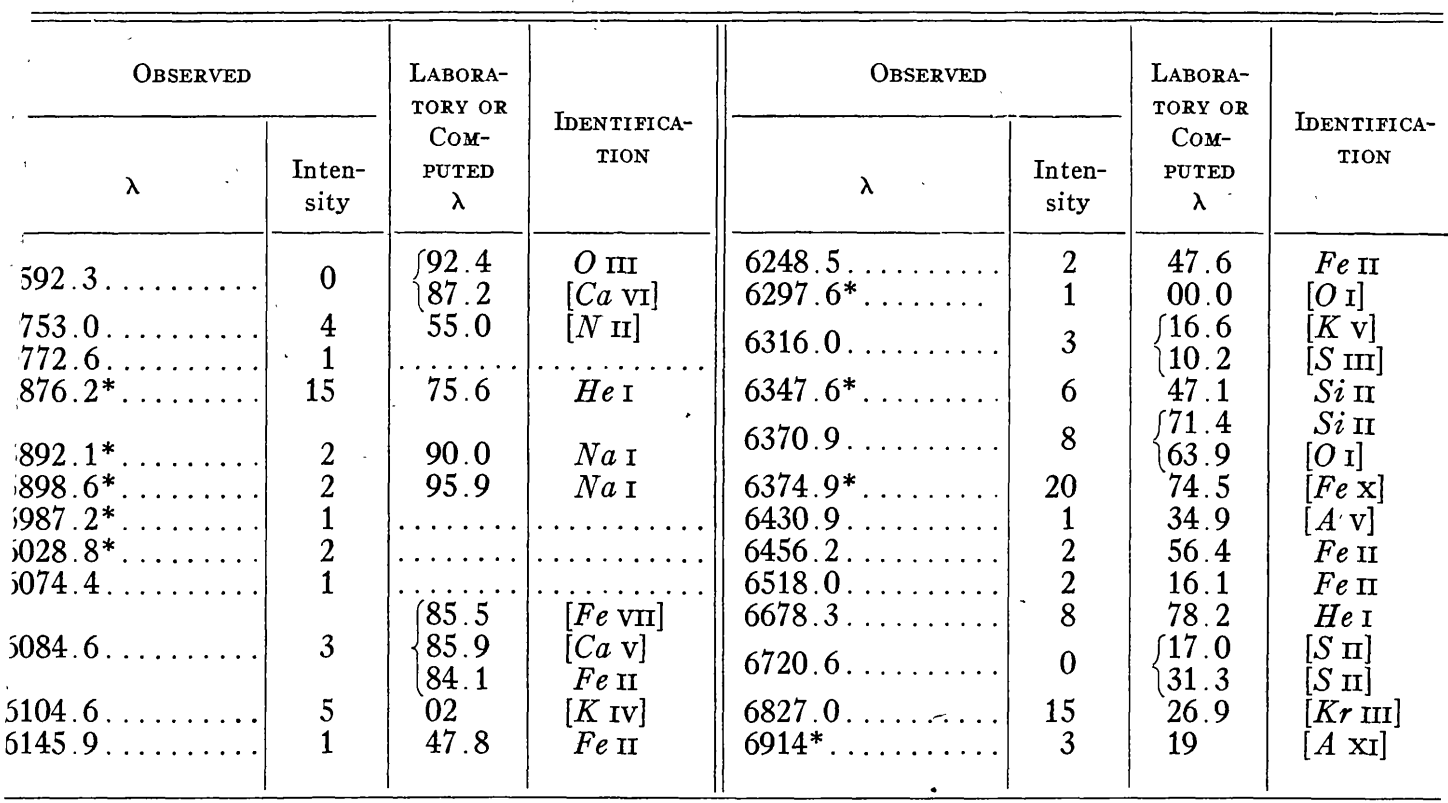

\begin{tabular}{|c|c|c|c|}
\hline$\lambda$ & Remarks & $\lambda$ & Remarks \\
\hline 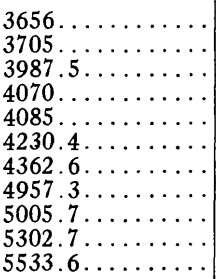 & $\begin{array}{l}\text { Wide } \\
\text { Wide } \\
\text { Corona } \\
\text { Wide } \\
\text { Corona } \\
\text { Corona } \\
\text { Wide band } \\
\text { N2 } \\
\text { N1 } \\
\text { Corona } \\
\text { Corona }\end{array}$ & 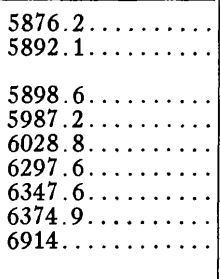 & $\begin{array}{l}\text { D3 } \\
\text { Violet comp. D1 and } \\
\quad \text { red comp. D2 } \\
\text { D1 red comp. } \\
\text { Wide } \\
\text { Wide } \\
\text { Wide } \\
\text { Wide } \\
\text { Corona } \\
\text { Wide }\end{array}$ \\
\hline
\end{tabular}

Table 1 is a list of the measured wave lengths and the approximate relative intensities of the lines. The possible identifications and the laboratory or computed wave lengths are in the third and fourth columns, the most probable identification or the largest contributor to a blend being given first.

The observed wave lengths are corrected for the earth's orbital motion but not for the radial velocity of the star itself. The hydrogen lines are present in considerable strength but have not been included in the table.

The measured wave lengths are compiled from the results from 12 spectrograms. The lines of wave length shorter than $\lambda 3850$ occur on a single spectrogram (dispersion 160 $\mathrm{A} / \mathrm{mm}$ at $\mathrm{K}$ ) obtained on October 27 . The longer wave lengths are derived from several plates with dispersions of from 40 to $120 \mathrm{~A} / \mathrm{mm}$ at $H \gamma$.

Most of the unblended bright lines are comparatively sharp and easily measured on good plates, but wave lengths cannot be measured closer than a few angstrom units with the dispersion employed.

\section{NEW IDENTIFICATIONS}

1. $[A \mathrm{xI}]$. - The line observed at $\lambda 6914$ is probably the $[A \mathrm{xI}]$ transition ${ }^{3} \mathrm{P}_{1}-{ }^{3} \mathrm{P}_{2}$, whose wave length was estimated by Edlén ${ }^{4}$ at $\lambda 6919$ with an uncertainty of a few angstrom units. Other lines falling in the same neighborhood $-\lambda 6911.05[N i \mathrm{II}], \lambda 6906.1$

${ }^{4}$ Zs.f. Ap., 22, 30, 1942; Ap. J.; 98, 127, 1943. 
[Cr IV], and $\lambda 6915.6[\mathrm{Cr}$ IV] — cannot be important contributors. Moreoyer, the $[A$ line $\lambda 5536$ is fairly strong, making the identification of $[A \mathrm{xI}]$ quite probable.

2. $[K r$ III] . - The strong line observed at $\lambda 6827$ (shown midway between the two e treme right-hand comparison lines in Plate IXe in the Publications of the Astronomic Society of the Pacific, 57, facing p. 173, 1945) agrees in wave length with the strong $\epsilon$ member of the forbidden transition ${ }^{3} \mathrm{P}-{ }^{1} \mathrm{D}$ of $K r \mathrm{III}$, and no other identification seer possible. The only other [ $K r \mathrm{III}]$ line $\lambda 9902.2$ is four times weaker than $\lambda 6827$. In $\mathrm{t}$ relatively cool layers where atoms may be in the doubly ionized state, the low excitatic potential of 1.81 volts of $[K r \mathrm{III}]$ favors the intensity of the [Kr III] line relative to oth forbidden lines, such as those of [Ne III] (E.P., 3.19 volts). Thus the identification $[K r \mathrm{III}]$ appears entirely satisfactory, and this conclusion is supported by the identific tion of the strong line $\lambda 5536$ with $[A \mathrm{x}]$ and the line $\lambda 6914$ with $[A \mathrm{xI}]$. The identi cation of argon and krypton lines indicates that conditions are favorable for the emissic of these lines, as well as for neon lines which have previously been found in a wide varie of celestial sources.

The ${ }^{3} \mathrm{P}-{ }^{1} \mathrm{D}_{2} \lambda 5846.3[\mathrm{Xe} \mathrm{III}]$ line of the heavier noble gas has been observed in tl Orion nebula, but in RS Ophiuchi the emission is extremely weak, if present.

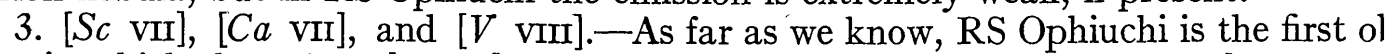
ject in which the $\lambda 4823$ [Sc VII] line and the blend of [ $\mathrm{Ca}$ VII] and [ $\mathrm{V}$ VIII] at $\lambda 36 \varepsilon$ have been observed; $\lambda 3686.3$ is the strongest [ $V \mathrm{VIII}]$ line; $\lambda 3688$ [Ca VII] is a ${ }^{1} \mathrm{D}-^{1}$ transition of relatively high probability.

\section{PHYSICAL CONSIDERATIONS}

A comparison of the intensities of the $[F e \mathrm{x}]$ and $[F e \mathrm{xIV}]$ lines shows that the excit: tion in the layers of RS Ophiuchi, where the lines of highly ionized atoms originate, definitely lower than in most regions of the solar corona, where the coronal lines ar emitted. RS Ophiuchi reveals several stages of ionization of iron and other atoms lowe than $\mathrm{x}$. This indicates that the physical conditions in this star differ radically from thos in the solar corona, where no trace of $F e$ atoms with ionization lower than that of $[F e$ s is found. On the sun the physical conditions in the layers intermediate between th $[F e \mathrm{x}]$ regions and the chromosphere do not permit ions such as $F e$ vII to emit their fo] bidden lines. This absence of $[\mathrm{Fe} \mathrm{VII}]$ lines is essentially the result of de-excitation by co lisions of ions in the metastable state. The transition probabilities of the strongest. $[F$ VII] lines are $0.47 \mathrm{sec}^{-1}$ and $0.37 \mathrm{sec}^{-1}$, but the higher ionization stages have much great 6 values, $69 \mathrm{sec}^{-1}$ for $[F e \mathrm{x}], 60$ for [ $\left.F e \mathrm{xrv}\right]$, and 106 for $[A \mathrm{x}]$. Hence there is little poss bility of $[\mathrm{Fe}$ VII] emission in the denser regions of the deep inner corona. In RS Ophiuch however, the stratification must be radically different from that in the sun, and thos regions of the star's atmosphere of intermediate ionization, rich in ions such as $F e$ VI. must have a density sufficiently low to permit the emission of the forbidden lines.

Stratification of a type similar to that of RS Ophiuchi is found in the recurring Nov (T) Pyxidis and perhaps should be looked for at certain times in other novae. The rela tive intensities of $[F e \mathrm{x}]$ lines with respect to those of $[F e \mathrm{XIV}]$ show that, in the region where the coronal lines are emitted, the ionization is higher in $T$ Pyxidis than in $R$ Ophiuchi, while lower density of the layers emitting $[F e$ VII] is probably the reason tha the intensity of $[F e \mathrm{VII}]$ relative to $[F e \mathrm{x}]$ and $[F e$ xIV] is greater in T Pyxidis than in $\mathrm{R}:$ Ophiuchi.

We are greatly indebted' to Dr. W. S. Adams for his kind co-operation in allowing u to make use of his spectrograms and measurements in this study. 